\title{
MBE Growth of Graded Structures for Polarized Electron Emitters
}

\author{
Aaron Moy, ${ }^{a}$ T. Maruyama, ${ }^{b}$ F. Zhou ${ }^{b}$ and A. Brachmann ${ }^{b}$ \\ ${ }^{a}$ SVT Associates, Eden Prairie, $M N$. \\ ${ }^{b}$ SLAC National Accelerator Laboratory, Menlo Park, CA
}

\begin{abstract}
SVT Associates, in collaboration with SLAC, have investigated two novel photocathode design concepts in an effort to increase polarization and quantum efficiency. $\mathrm{AlGaAsSb} / \mathrm{GaAs}$ superlattice photocathodes were fabricated to explore the effect of antimony on device operation. In the second approach, an internal electrical field was created within the superlattice active layer by varying the aluminum composition in $\mathrm{AlGaAs} / \mathrm{GaAs}$. A $25 \%$ increase in quantum efficiency as a result of the gradient was observed.
\end{abstract}

Keywords: Polarized Photocathode

PACS: 29.25.Bx, 29.27. $\mathrm{Hj}$

\section{INTRODUCTION}

Polarized electrons have been essential for high-energy parity-violating experiments and measurements of nucleon spin structure, and polarized electron beams will be required for all future linear colliders. Polarized electrons are readily produced by GaAs photocathode sources. When a circularly polarized laser beam tuned to the bandgap minimum is directed to the negative-electron-affinity (NEA) surface of a GaAs crystal, longitudinally polarized electrons are emitted into vacuum. The electron polarization is easily reversed by reversing the laser polarization. The theoretical maximum polarization of 50\% for natural GaAs was first exceeded in 1991 using the lattice mismatch of a thin InGaAs layer epitaxially grown over a GaAs substrate to generate a strain in the former that broke the natural degeneracy between the heavy- and light-hole valence bands. ${ }^{1}$

In a previous collaboration between SVT Associates and SLAC, photocathodes were developed based on $\mathrm{GaAs}_{0.64} \mathrm{P}_{0.36} / \mathrm{GaAs}$ superlattice. These devices demonstrated polarizations as high as $86 \%$, with quantum efficiencies exceeding $1 \%{ }^{2}$ Two known factors limit the polarization of these cathodes: 1) a limited band splitting; and 2) a relaxation of the strain in the surface epilayer since the 10-nm critical thickness for the $1 \%$ lattice-mismatch is exceeded.

In an effort to advance photocathode operating characteristics, two different device structures were examined. The first design employed $\mathrm{Al}_{\mathrm{x}} \mathrm{Ga}_{1-\mathrm{x}} \mathrm{As}_{\mathrm{y}} \mathrm{Sb}_{1-\mathrm{y}}$ material to reduce depolarization. A second design employed an $\mathrm{Al}_{\mathrm{x}} \mathrm{Ga}_{1-\mathrm{x}} \mathrm{As} / \mathrm{GaAs}$ superlattice where the aluminum content was varied during the growth. This graded $\mathrm{Al}_{x} \mathrm{Ga}_{1-\mathrm{x}} \mathrm{As}$ 
thus created an internal electrical field that would serve to accelerate electrons to the surface, increasing quantum efficiency.

\section{ANTIMONIDE-BASED PHOTOCATHODE STRUCTURES}

To address material-specific spin-depolarization and increase the photocathode robustness which is essential for an RF gun, a strained superlattice structure based on GaAs and $\mathrm{Al}_{\mathrm{x}} \mathrm{Ga}_{1-\mathrm{x}} \mathrm{As}_{\mathrm{y}} \mathrm{Sb}_{1-\mathrm{y}}$ was explored. In this structure, the $\mathrm{Al}_{\mathrm{x}} \mathrm{Ga}_{1-\mathrm{x}} \mathrm{As}_{\mathrm{y}} \mathrm{Sb}_{1-\mathrm{y}}$ layers serve as energy barriers. The GaAs layers are wells and are strained. The addition of antimony ( $\mathrm{Sb}$ ) to the material was thought to have two benefits. Firstly, antimony has 3 orders of magnitude lower diffusivity than gallium and 2 orders of magnitude lower diffusivity than arsenic. ${ }^{3}$ Diffusion is one of the main reasons for structural and surface decomposition of cathodes, especially during heat cleaning and surface bombardment.

Secondly, antimony has higher spin orbit coupling than As, so the effect of spin orbit coupling on depolarization can be studied. The increased spin orbit coupling allows structures with larger HH-LH separation, which can increase the polarization of the photocathode effectively. Thus it is believed that antimony-based photocathodes could play an important role in spin polarized RF guns because they can combine high polarization and high robustness in poor vacuum environment

Figure 1 displays a diagram of the AlGaAsSb/GaAs superlattice structure explored. Polarized emission continues from the GaAs layers in this structure, with the strain and barrier energies being defined by the $\mathrm{Al}_{\mathrm{x}} \mathrm{Ga}_{1-\mathrm{x}} \mathrm{As}_{\mathrm{y}} \mathrm{Sb}_{1-\mathrm{y}}$ alloy composition. The addition of antimony ( $\mathrm{Sb}$ ) atoms in the material growth was a novel approach to the design of photocathodes with polarized electron emission.

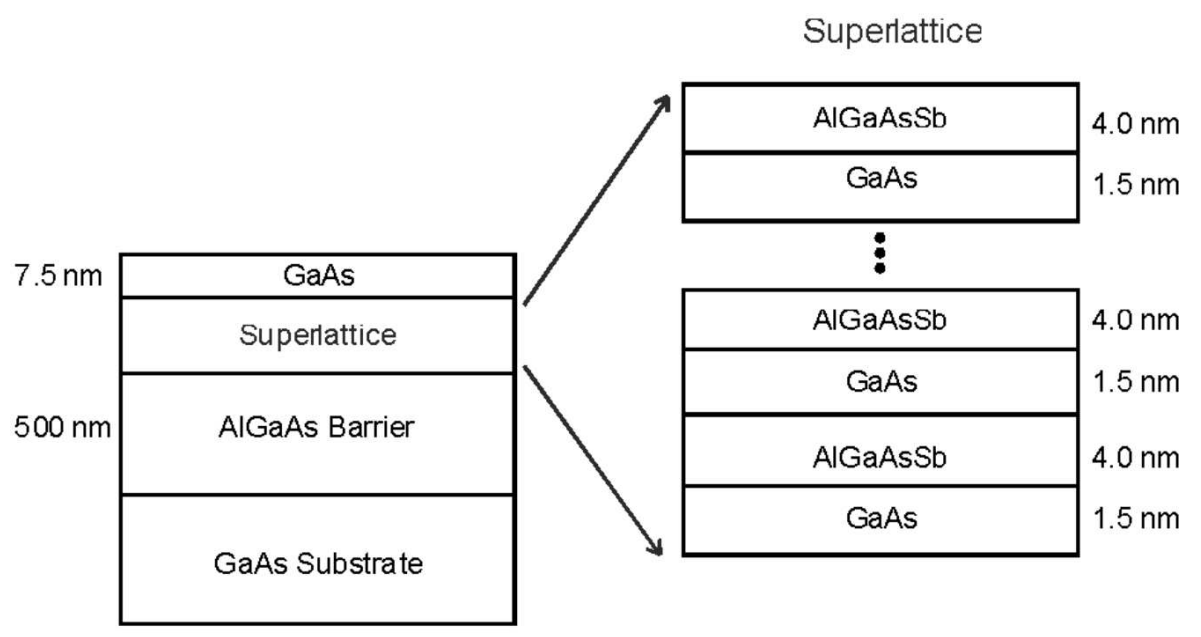

FIGURE 1. Diagram of the basic photocathode structure studied utilizing a superlattice consisting of $\mathrm{GaAs}$ wells and $\mathrm{AlGaAsSb}$ barriers. 
The spin orbit (SO) coupling is more pronounced in superlattice structures due to heterointerface asymmetries. A 10-15nm GaAs layer of high p- doping $\left(5 \times 19 \mathrm{~cm}^{-3}\right)$ at the cathode surface is desirable in order to achieve NEA reliably. The SL structure should have low p-doping $\left(\sim 1 \times 17 \mathrm{~cm}^{-3}\right)$ in order to increase mobility and avoid depolarization due to scattering. The increased doping at the surface results in an elevated CB at the surface that can be compensated by the SL miniband as long as the conduction miniband is higher that the conduction band level at the surface. The entire structure was grown on a GaAs substrate with an $\mathrm{AlGaAs}$ energy barrier layer.

In principle, the $\mathrm{Al}_{x} \mathrm{Ga}_{1-\mathrm{x}} \mathrm{As}_{\mathrm{y}} \mathrm{Sb}_{1-\mathrm{y}}$ quaternary alloy provides design flexibility. The percentage of $\mathrm{Sb}$ can be used to experiment with the effect of spin orbit coupling and band lineup and the $\mathrm{Al}$ percentage can be used to control the band offsets and LH, HH valence band splitting.

Three device structures were grown on 2" diameter GaAs substrates by molecular beam epitaxy (MBE). The structures all utilized $\mathrm{Al}_{x} \mathrm{Ga}_{1-\mathrm{x}} \mathrm{As}_{0.82} \mathrm{Sb}_{0.18}$ alloy in the superlattice, where the aluminum composition was changed between the samples being $\mathrm{x}=5,10$ or $15 \%$.

\section{X-Ray of $10 \%$ Al Photocathode structure}
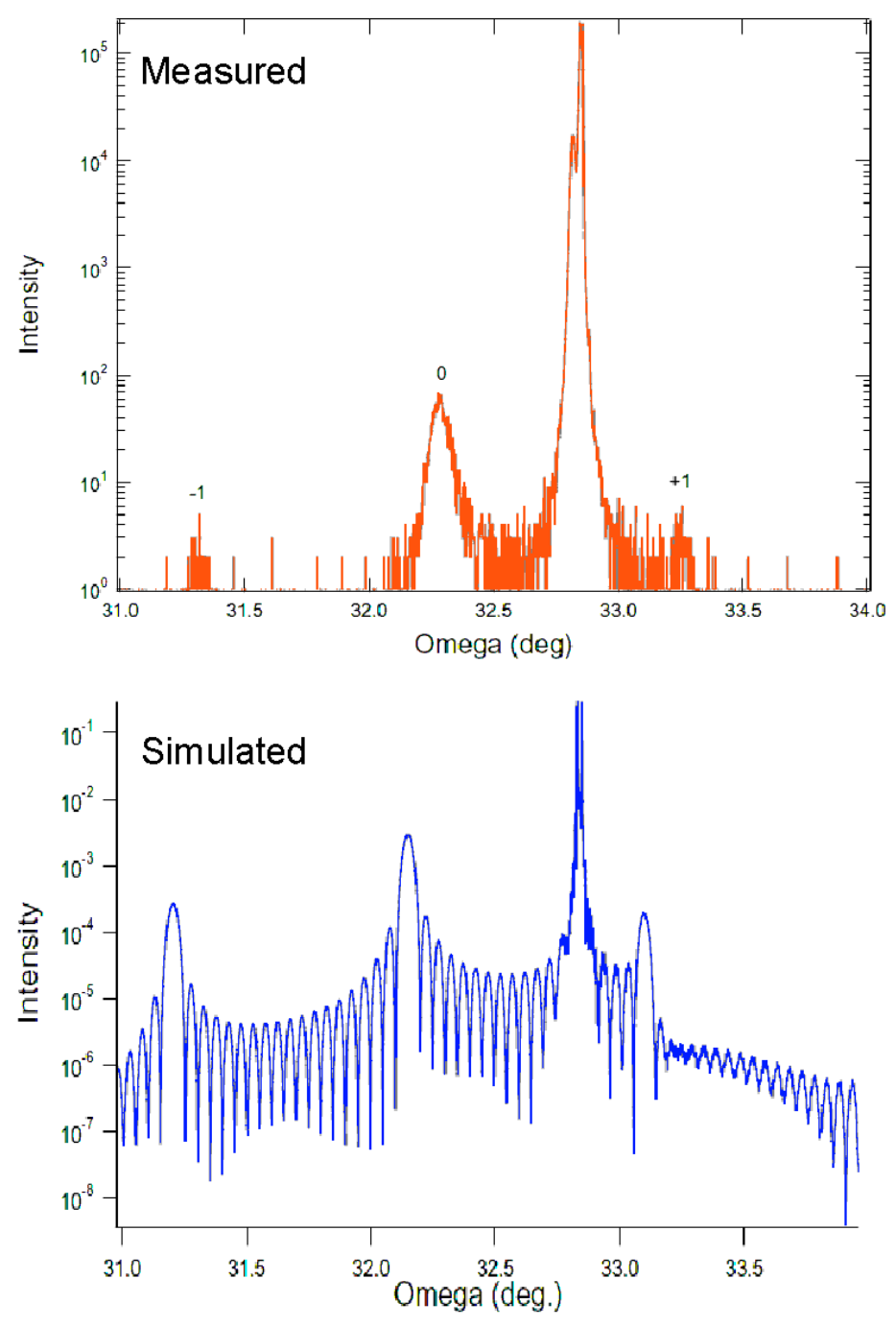

Figure 2.

$\mathrm{X}$-ray diffraction measurement and simulation of $\mathrm{AlGaAsSb} / \mathrm{GaAs}$ superlattice photocathode structure. The simulation assumes $100 \%$ strain. 
X_ray diffraction was performed on the device wafer to confirm and measure the superlattice (Fig. 2). A simulation of the structure was also calculated assuming 100\% strain in the structure. The superlattice peaks are observed in the measured data, as expected from the superlattice layers. However, the location of the sideband peaks is slightly shifted, indicating that some relaxation in the material may have occurred.

The wafers were sent to SLAC where they were cut into dies and cesium-activated. Prior to installation in the test system, the sample is degreased in a boiling solution of trichloroethane. After the protective oxide layer is removed in ammonium hydroxide, the sample is rinsed in distilled water and methanol. The cathode activation method used to obtain a negative-electron-affinity (NEA) surface consists of heat cleaning to $600^{\circ} \mathrm{C}$ for 1 hour, cool-down for an hour, followed by application of cesium until the photo-yield peaks, and then cesium and nitrogen-trifluoride co-deposition until the photo-yield is again maximized. The heat-cleaning temperature is monitored via an infrared pyrometer. The cathode is activated while monitoring the photo-yield with a white light and a $670 \mathrm{~nm}$ diode laser. Once a red response is observed from the diode laser, the white light is turned off and the diode laser is used to complete the activation. The absolute $\mathrm{QE}$ is measured using the diode laser at a photon wavelength of $670 \mathrm{~nm}$. A tungsten lamp and a monochrometer are used to measure the relative $\mathrm{QE}$ as a function of photon wavelength, and these measurements are then normalized to the diode laser measurement at $670 \mathrm{~nm}$

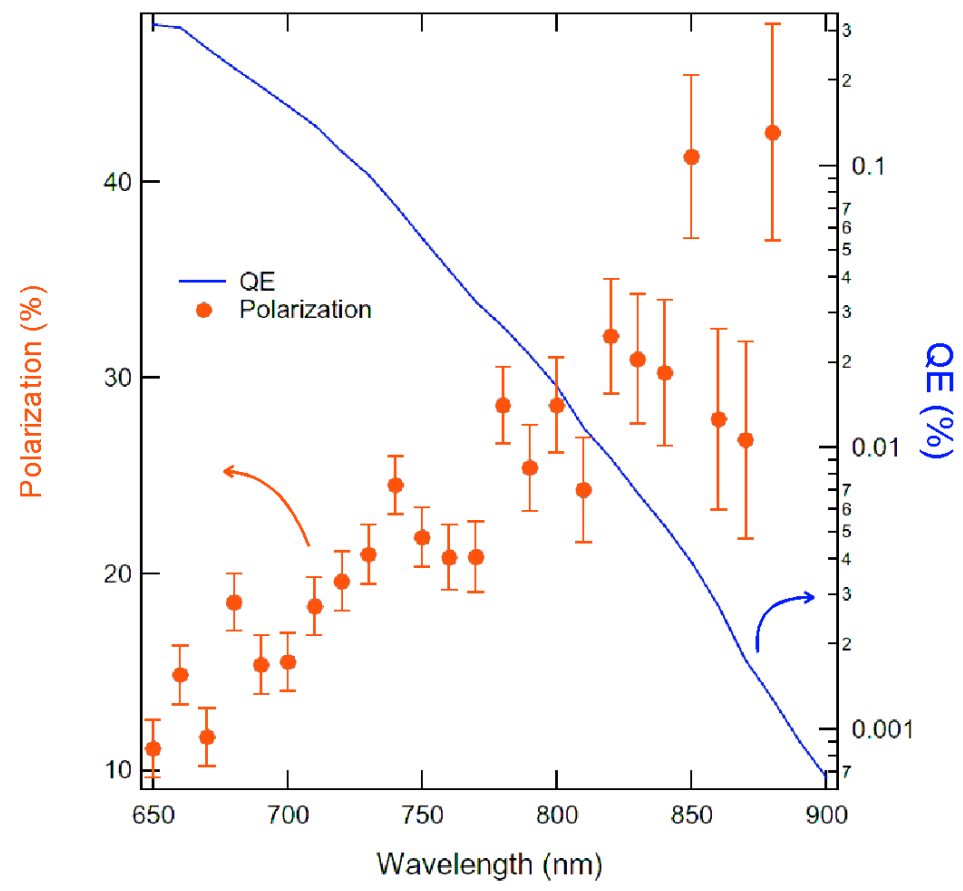

Figure 3. Quantum efficiency and polarization measurements from the AlGaAsSb/GaAs superlattice photocathodes with $5 \%$ aluminum composition.

Measurement of the quantum efficiency $(\mathrm{QE})$ from the $10 \%$ aluminum sample was very low, $\sim 0.01 \%$ at $650 \mathrm{~nm}$ excitation wavelength. Such emission was too low to measure polarization. The 5 and $15 \%$ aluminum composition samples did yield 
higher currents, with QE of $0.2 \%$ and $0.1 \%$, respectively. Polarization was measured from the $5 \%$ aluminum sample, but demonstrated polarization of less than $50 \%$ (Fig. 3).

Unfortunately, the device results for antimony-based $\mathrm{Al}_{\mathrm{x}} \mathrm{Ga}_{1-\mathrm{x}} \mathrm{As}_{0.82} \mathrm{Sb}_{0.18} / \mathrm{GaAs}$ photocathode were not as anticipated. Both quantum efficiency and polarization values were very low. This could be a result of this superlattice design and not wafer quality. The hypothesis is that the confinement energy around the quantum wells was too high, trapping electrons inside and hampering their contribution to the photoemission. Another possibility is that the compressively-strained AlGaAsSb layers, which are normally grown at temperatures below $500{ }^{\circ} \mathrm{C}$, could have been damaged during thermal removal of surface native oxide at $600{ }^{\circ} \mathrm{C}$ before surface activation process.

\section{GRADED ALUMINUM GALLIUM ARSENIDE SUPERLATTICE PHOTOCATHODES}

Another approach to increasing quantum efficiency is to create an electrical field internal to the photocathode active layer to accelerate electrons toward the negative electron affinity (NEA) surface. Figure 4 displays the concept, where the conduction band of the superlattice has a sloped gradient field to influence electron flow, as opposed to a typical design where the barrier heights exist at a constant level. To achieve such a gradient, $\mathrm{Al}_{\mathrm{x}} \mathrm{Ga}_{1-\mathrm{x}} \mathrm{As}$ alloys were used as barriers. This $\mathrm{Al}_{\mathrm{x}} \mathrm{Ga}_{1-\mathrm{x}} \mathrm{As}$ material permitted wide latitude in changing the bandgap energy by varying the composition $\mathrm{x}$ during growth, without creating undo changes to the overall lattice constant.

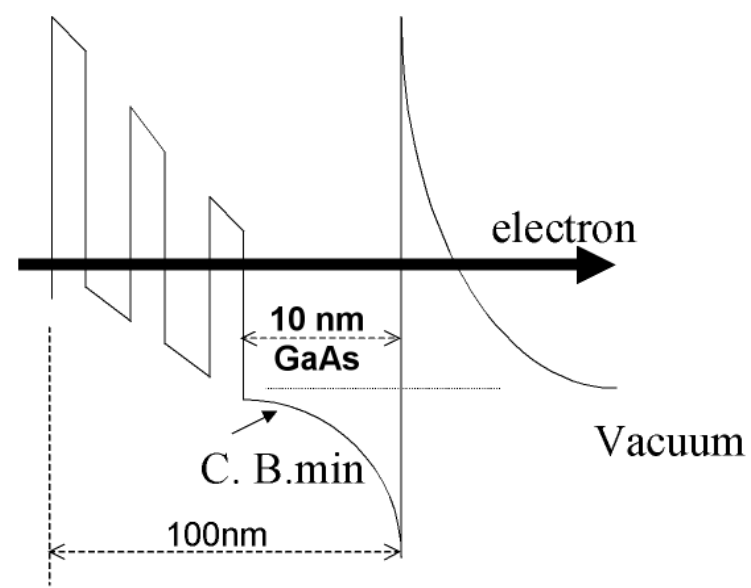

Figure 4. Diagram of the concept to create an accelerating field internal to the photocathode structure in order to drive electrons to the surface for increased quantum efficiency.

In the presence of such accelerating field, the electron transport time decreases by an order of magnitude to less than $1 \mathrm{ps}$ as it has been estimated by Monte Carlo 
simulations and verified experimentally. ${ }^{4,5,6}$ The decrease of the transport time by an order of magnitude not only increases the current density that is required for an RF gun by the same amount but it also increases the polarization. In the case of thin films $(<100 \mathrm{~nm})$ the polarization can increase by decreasing the transport time of the electrons and decreasing the total number of scattering events (equation 1). ${ }^{7}$

$$
\frac{P_{2}}{P_{1}}=\frac{1+\frac{\tau_{e m 1}}{\tau_{s 1}}}{1+\frac{\tau_{e m 1}}{\tau_{s 1}}\left(\frac{s_{2}}{s_{1}} \frac{\tau_{e m 2}}{\tau_{e m 1}}\right)}
$$

where:

$\tau_{\mathrm{em}}$ : time of electron emission in vacuum

P: electron polarization upon excitation with circularly polarized light s: total number of scattering events.

Based on the simple model of Equation (1), an increase of 5-10\% in polarization can be achieved with accelerating the electrons.

The main objective of these experiments was to demonstrate an internal bias using an $\mathrm{Al}_{\mathrm{x}} \mathrm{Ga}_{1-\mathrm{x}} \mathrm{As} / \mathrm{GaAs}$ superlattice structure. Figure 5 shows the reference sample layout, where the active layer consisted of 19 periods of superlattice layers, with 11 monolayers of $\mathrm{Al}_{\mathrm{x}} \mathrm{Ga}_{1-\mathrm{x}} \mathrm{As}$ and 7 monolayers of $\mathrm{GaAs}$ in each pair. For the reference sample, the aluminum composition $\mathrm{x}$ was held constant at 0.35 . An internal bias was introduced into subsequent photocathodes by varying the aluminum fraction, $\mathrm{x}$, throughout the active layer. By slightly varying the aluminum fraction between each pair through the superlattice, a graded field was created. This type of design, however, adds complexity to the epitaxial growth, as atomic mole fraction must be quickly and precisely varied.

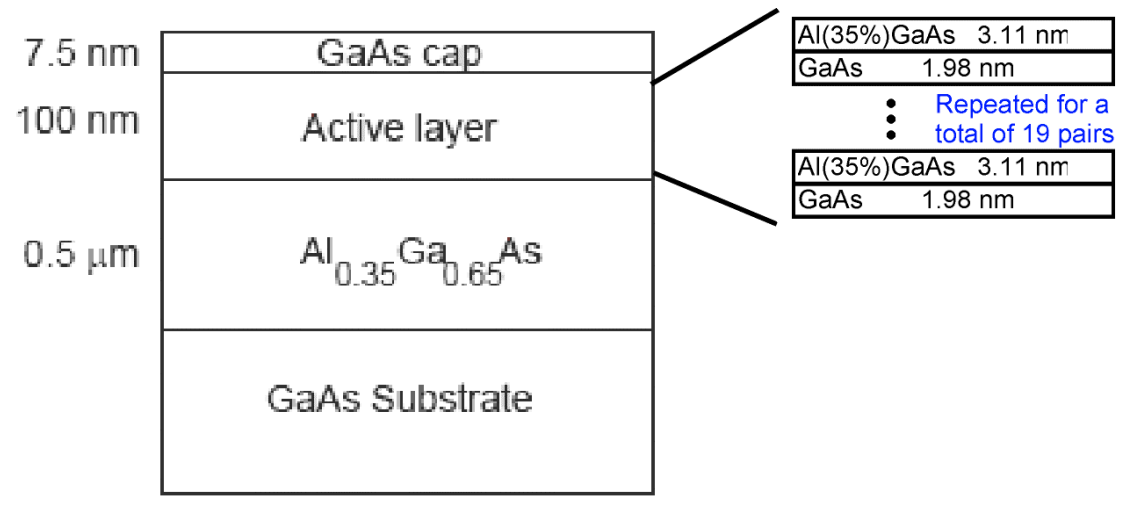

Figure 5. Schematic structure of the reference $\mathrm{AlGaAs} / \mathrm{GaAs}$ superlattice photocathode.

The superlattice band structure was studied using the $\mathbf{k} \cdot \mathbf{p}$ envelope function method. Figure 6(a) shows the mini-band energies as a function of the superlattice well width for a fixed barrier width ( 7 monolayers). The two highest energy valence bands correspond to the heavy- and light-hole mini-bands, and the maximum energy 
splitting was expected to be about $28 \mathrm{meV}$. Figure 6(b) shows the expected polarization and $\mathrm{QE}$ as a function of the excitation wavelength. The band gap wavelength was expected at $760 \mathrm{~nm}$.

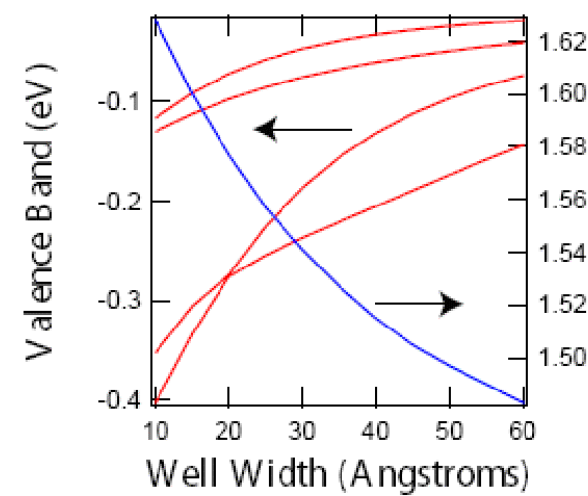

(a)

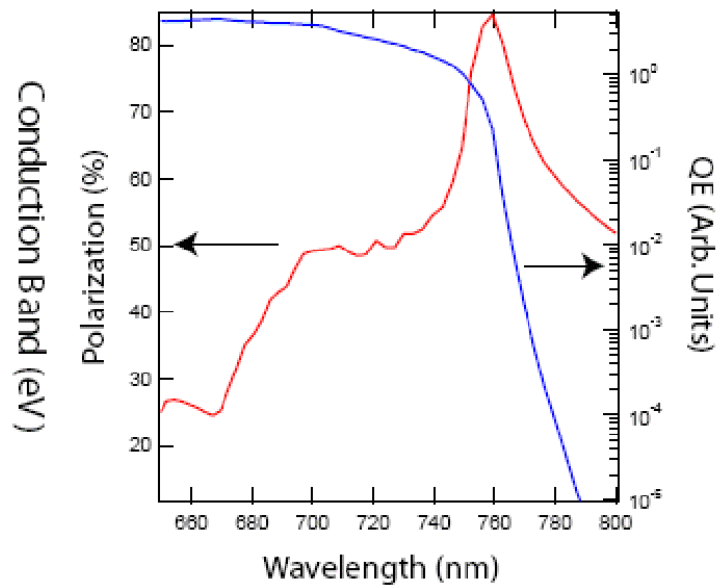

(b)

Figure 6. (a) Superlattice mini-band energies as a function of the well width. (b) Theoretical predictions for the polarization and QE.

Three wafers were grown with different aluminum grading values in the active layer: 1) no grading with $\mathrm{x}=0.35,2$ ) $10 \%$ grading from $\mathrm{x}=0.35$ to 0.25 , and 3) $20 \%$ grading from $x=0.35$ to 0.15 . Because of the thin amount of the active layer and the extremely low lattice mismatch between the $\mathrm{Al}$ and $\mathrm{Ga}$ atoms, the x-ray diffraction simulation predicted two weak, widely spaced satellite peaks (Fig. 7) for the superlattice. Actual measured x-ray data of the grown wafer was in close agreement with the simulation (Fig. 8).

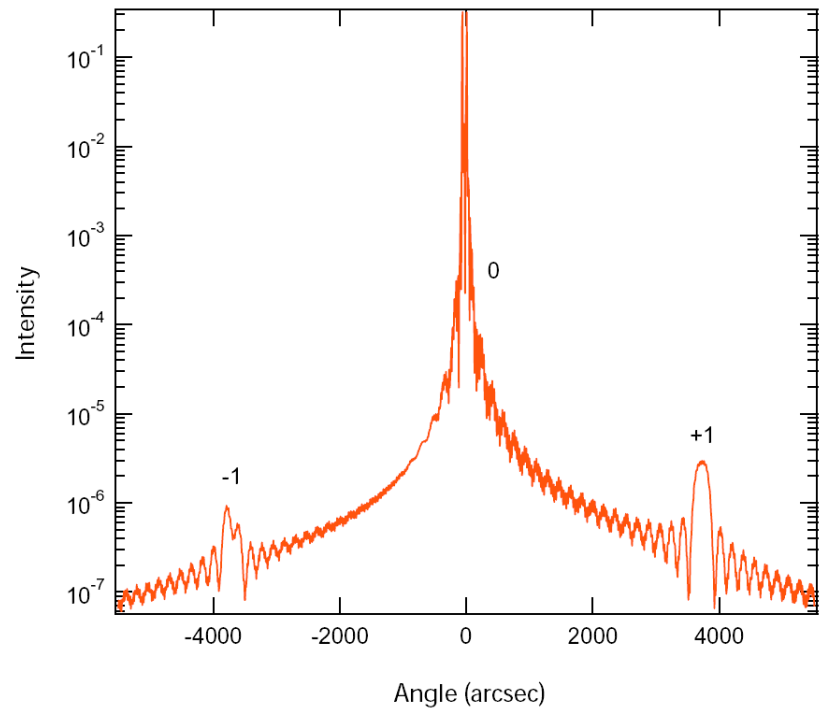

Figure 7. Simulation of anticipated x-ray diffraction curve based on the graded AlGaAs/GaAs superlattice structure. 


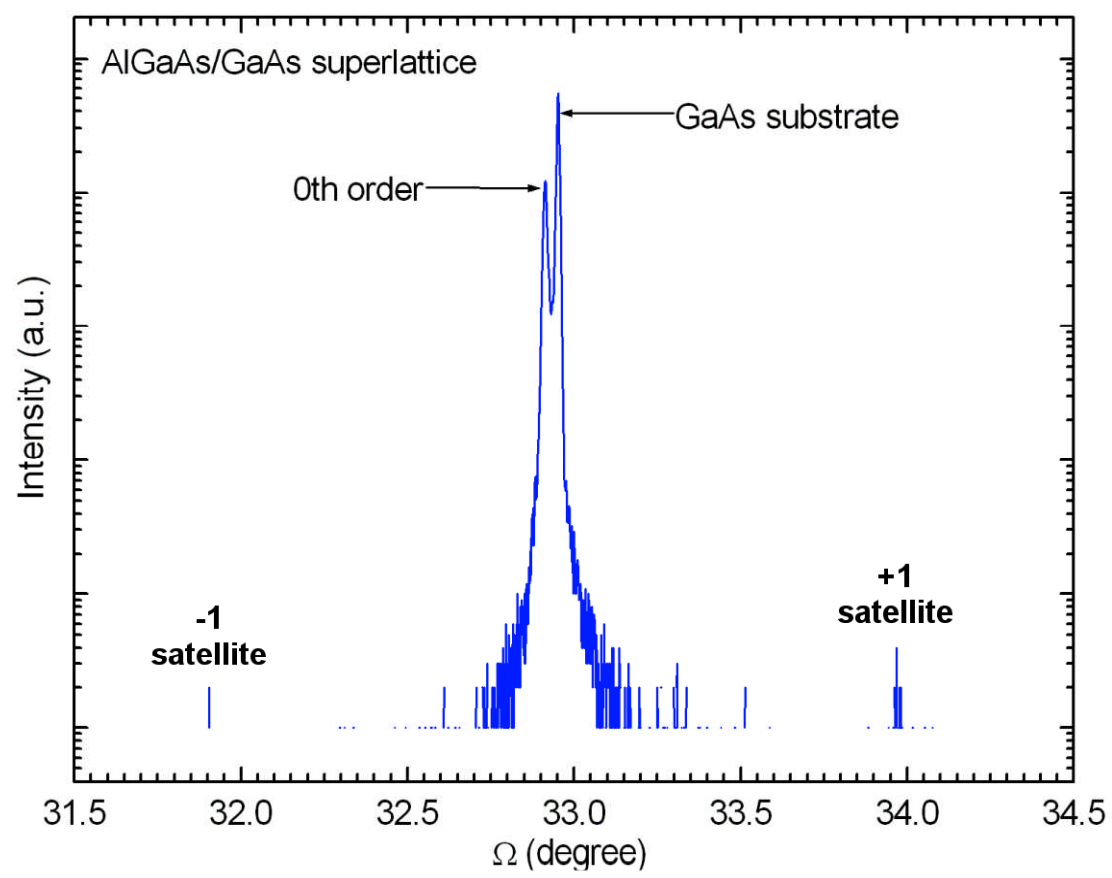

Figure 8. Measured x-ray diffraction curve of the grown AlGaAs/GaAs superlattice structure.

The photocathode wafers were cleaned and activated as described in the previous section. Figure 9 shows the QE at $650 \mathrm{~nm}$ for the three samples. As the internal bias is increased by increasing the aluminum grading, the QE increases as much as $25 \%$. This is the first demonstration that an electric field can be incorporated in the conduction band and a $\mathrm{QE}$ enhancement is possible.

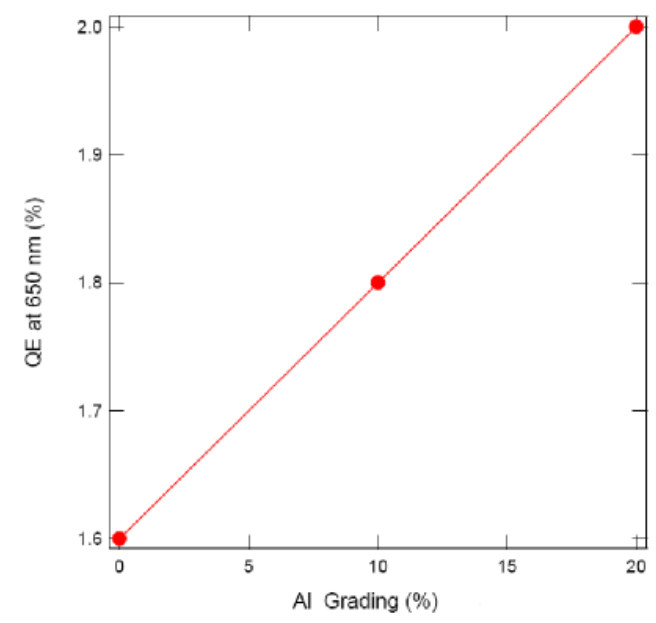

Figure 9. QE at $650 \mathrm{~nm}$ as a function of the aluminum grading in $\mathrm{AlGaAs} / \mathrm{GaAs}$ superlattice photocathodes. 
Figure 10 shows QE and polarization as a function of wavelength for the three samples tested. As the aluminum grading was increased, the peak polarization decreased. Furthermore, the wavelength dependence of QE became steeper, eliminating the QE gain in the short wavelength region. This is a consequence of the structural change in the superlattice active layer. As the aluminum fraction is decreased, the effective bandgap energy gets smaller and the heavy- and light-hole splitting is reduced.

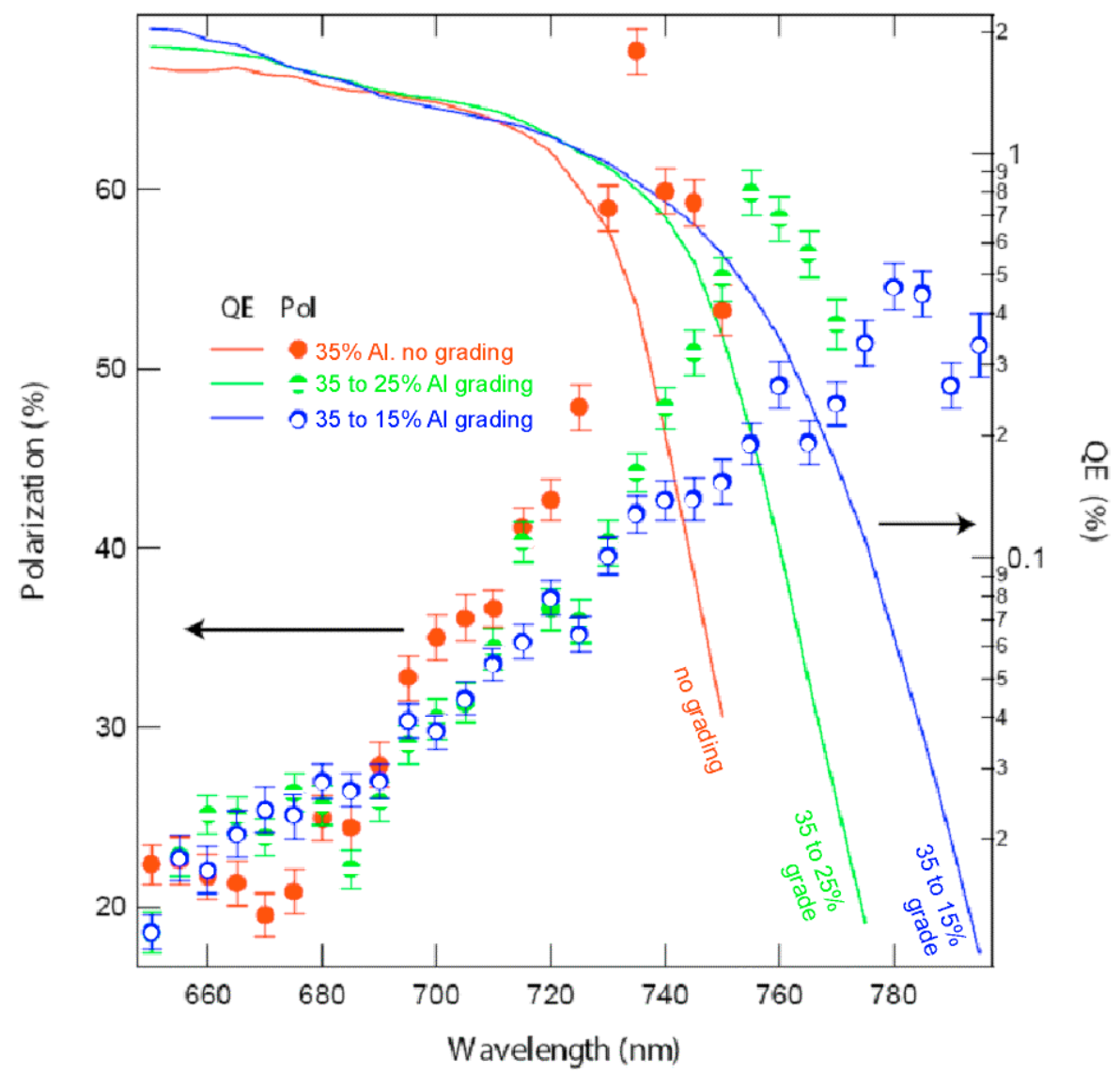

Figure 10. Polarization and QE as a function of the excitation wavelength for three AlGaAs/GaAs superlattice photocathodes with internal gradient fields.

\section{CONCLUSION}

Two novel device structures were studied to improve the operating characteristics of photocathodes with polarized electron emission. $\mathrm{Al}_{\mathrm{x}} \mathrm{Ga}_{1-\mathrm{x}} \mathrm{As}_{\mathrm{y}} \mathrm{Sb}_{1-\mathrm{y}} / \mathrm{GaAs}$ superlattices were created to take advantage of the lower diffusivity and higher spin orbit coupling of antimony atoms. These photocathodes in this instance yielded low quantum efficiency, attributed to the extremely deep well energy. Further investigation can continue in the future with a modified design.

The second approach was to create a superlattice with a self-generated internal electric field to accelerate electrons toward the photocathode surface. This was accomplished by varying the aluminum composition in a $\mathrm{Al}_{\mathrm{x}} \mathrm{Ga}_{1-\mathrm{x}} \mathrm{As} / \mathrm{GaAs}$ 
superlattice. Quantum efficiency was observed to increase by up to $25 \%$ compared to a control sample that lacked an internally graded field. The low strain in $\mathrm{Al}_{\mathrm{x}} \mathrm{Ga}_{1-\mathrm{x}} \mathrm{As} / \mathrm{GaAs}$ materials resulted in mild polarization values, but the internal gradient concept can be applied to other high polarization photocathodes.

\section{ACKNOWLEDGMENTS}

This research was supported by Small Business Innovation Research (SBIR) grants

from the US Dept. of Energy under contracts \#DE-FG02-07ER86329 and \#DE-FG0207ER86330.

\section{REFERENCES}

1. T. Maruyama, E.L. Garwin, R. Prepost, G.H. Zapalac, J.S. Smith, and J.D. Walker, Phys. Rev. Lett. 66, 2376 (1991).

2. T. Maruyama, D.-A. Luh, A. Brachmann, J.E. Clendenin, E.L. Garwin, S. Harvey, J. Jian, R.E. Kirby, C.Y. Prescott, R. Prepost, and A.M. Moy, Appl. Phys. Lett. 85, 2640 (2004).

3. H. Bracht, S.P. Nicols, W. Walukiewitz, J.P. Silveira, F. Briones, E.E. Haller, "Large disparity between gallium and antimony self diffusion in gallium antimonide", Nature, 408, 69 (2000).

4. Schuler,J., et al., Polarized sources conf., Nagoya 2000.

5. K. Ioakeimidi, et al., "Photoelectronic analog-to-digital conversion: sampling and quantizing at $100 \mathrm{Gs} / \mathrm{s}$ ", IEEE Trans. Microwave Theory and Techniques, Vol. 53, Issue 1, Jan. 2005.

6. K. Ioakeimidi et. al., "Sub-picosecond jitter in picosecond electron bunches", J. Vac. Sci. Technol. B, Vol. 23, (2005).

7. K. Ioakeimidi et al., "Transport Mechanisms in Spin Polarized Semiconductor Superlattices", SPIN 2006, 1-7 October 2006, Kyoto, Japan. 